\title{
Further Results on Extended Delivery Time for Secondary Packet Transmission
}

\author{
Muneer Usman, Member, IEEE, Hong-Chuan Yang, Senior Member, IEEE, \\ Mohamed-Slim Alouini, Fellow, IEEE
}

\begin{abstract}
Cognitive radio transceiver can opportunistically access the underutilized spectrum resource of primary systems for new wireless services. With interweave cognitive implementation, secondary transmission may be interrupted by primary transmission. To facilitate the packet delay analysis of such secondary transmission, we study the extended delivery time of secondary packet transmission. In particular, we derive the exact distribution function of extended delivery time of a fixed-size secondary packet with non-work-preserving strategy, where interrupted packets must be repeated. We also analyze the effect of imperfect periodic sensing i.e. the secondary user periodically senses the spectrum for availability, with a chance of missing an available channel on a certain sensing attempt. These results complement previous work on work-preserving strategy with perfect sensing. Selected numerical and simulation results are presented for verifying the mathematical formulation.
\end{abstract}

Index Terms

Cognitive radio, spectrum access, traffic model, primary user, secondary user, imperfect sensing, false alarm, Markov chain.

\section{INTRODUCTION}

Radio spectrum scarcity is one of the most serious problems nowadays faced by the wireless communications industry. By exploiting temporal/spatial spectrum opportunities over the existing licensed frequency bands, cognitive radio is a promising solution to this problem [1]-[8]. Different techniques exist for opportunistic spectrum access (OSA). In underlay cognitive radio

This paper was presented in part at International Zurich Seminar on Communications, 2016. 
implementation, the primary and secondary users simultaneously access the same spectrum, with a constraint on the interference that secondary user (SU) may cause to primary transmission. With interweave cognitive radio implementation [9], the secondary user (SU) can access the channel only when the channel is not used by primary user (PU) and must vacate the occupied channel when the PU appears. Spectrum handoff procedures are adapted for returning the radio channel to the PU and then re-accessing that channel or another channel later to complete the transmission. The secondary transmission of a given amount of data may involve multiple transmission attempts, and hence, extra transmission delay. When the secondary transmission is interrupted by PU activities, the secondary system can adopt either non-work-preserving strategy, where interrupted packets transmission must be repeated [10], or work-preserving strategy, where the secondary transmission can continue from the point where it was interrupted, without wasting the previous transmission [11]. The total time required for the SU to complete a given packet transmission will include the waiting periods before accessing the channel, the periods of the wasted transmissions in case of non-work-preserving strategy, and the time used for the final successful transmission. In our previous work [12], we carried out a thorough statistical analysis on the resulting extended delivery time (EDT) [10] of secondary packets i.e. packets transmitted by the secondary user) for work-preserving strategy with perfect sensing. Here, we complement previous work by considering non-work-preserving strategy and imperfect sensing cases.

\section{A. Previous Work}

There has been a continuing interest in the delay and throughput analysis for secondary systems, especially for underlay implementation. [13] analyzes the delay performance of a point-to-multipoint secondary network, which concurrently shares the spectrum with a pointto-multipoint primary network in the underlay fashion, under Nakagami- $m$ fading. The packet transmission time for secondary packets under PU interference is investigated in [14], where multiple secondary users are simultaneously using the channel. [15] examines the probability density function (PDF) and cumulative distribution function (CDF) of secondary packet transmission time in underlay cognitive system. [16] investigates the M/G/1 queue performance of the secondary packets under the PU outage constraint.

For interweave implementation strategy, [17] discusses the average service time for the SU in a single transmission slot and the average waiting time, i.e. the time the SU has to wait for the 
channel to become available, assuming general primary traffic model. A probability distribution for the service time available to the SU during a fixed period of time was derived in [18]. [19] derives expressions for average delay for continuous and periodic sensing. [20] studies the probability of successful data transmission in a cooperative wireless communication scenario with hard delay constraints. A queuing analysis for secondary users dynamically accessing spectrum in cognitive radio systems was carried out in [21], where the authors analyze the maximum possible packet arrival rate the secondary system can support. [22] proposes a dynamic channel selection approach to minimize the delay for secondary users in a pre-emptive resume priority M/G/1 queuing network. End-to-end performance of a cognitive radio network has been analyzed in terms of quality of service parameters in [23].

The concept of EDT was first used in [10] to derive bounds on the throughput and delay performance of secondary users in cognitive scenario. The expected EDT of a packet for a cognitive radio network with multiple channels and users has been calculated in [11]. In particular, the EDT statistics also depend on channel sensing strategy as well as packet transmission strategy used, either work-preserving strategy or non-work-preserving strategy, when the secondary transmission is interrupted by PU activities.

For the purpose of cognitive analysis, a number of sensing strategies can be considered. With the ideal continuous sensing, the SU will continuously sense for the channel availability, while PU is transmitting. In the practical scenario of periodic sensing, the SU will sense the channel periodically [17]. [17] focuses on a single secondary transmission slot while discussing periodic sensing. Design of periodic sensing parameters has been discussed in [24], [25]. A framework of Markov decision processes is presented by [8] to derive the optimal policy for channel access under periodic sensing assumption. Considering the more practical scenario of imperfect periodic sensing, the probability of false alarm and miss detection can be introduced into the analysis. Symbol error rate performance with imperfect sensing has been analyzed in [26]. [27] analyzes the throughput of a secondary system using a MAC protocol with perfect and imperfect sensing. A sensing order selection scheme for imperfect sensing has been discussed in [28]. [29] proposes a framework to optimize the EDT with channel sensing errors. [30] tries to find the optimal trade-off between spectrum sensing power consumption, SU throughput, and interference, with imperfect sensing. The effect of spectrum sensing errors on cognitive transmission has been analyzed in [31]. The capacity of a secondary cognitive channel with sensing error is analyzed 
in [32]. To the best of our knowledge, there has been little previous work on delay analysis for periodic spectrum sensing.

In our previous work [12], we carried out a thorough statistical analysis on the EDT of secondary packet transmission with work-preserving strategy, and then applied these results to the secondary queuing analysis. Typically, work-preserving packet transmission requires packets to be coded with certain rateless codes such as fountain codes, which may not be available in the secondary system.

\section{B. Contribution}

In this paper, we investigate the statistical characteristics of the EDT with non-work-preserving strategy, and apply them to evaluate the delay performance of secondary transmission. Analysis with non-work-preserving strategy is, in general, more challenging as the transmission of a secondary packet will involve an interweaved sequence of wasted transmission slots and waiting time slots, both of which can have random time duration, followed by the final successful transmission slot. We consider the scenarios of perfect periodic sensing and imperfect periodic sensing, taking the probability of false alarm, i.e. sensing a free channel to be busy, into account. We also extend our previous work by analyzing the effect of imperfect periodic sensing with work-preserving strategy.

For each of these scenarios, we derive the exact statistics of the EDT for secondary packet transmission in terms of probability density function (PDF). These results can be applied to secondary queuing analysis for high-traffic applications in a similar manner as in [12].

The major novel contributions of this paper can be summarized as follows:

1) Complete and exact statistics of the EDT for secondary packet transmission with non-workpreserving strategy and periodic sensing. To the best of our knowledge, the complete and exact statistics of the EDT for non-work-preserving strategy has not been investigated in literature.

2) Complete and exact statistics of the EDT for both transmission strategies with imperfect periodic sensing. To the best of our knowledge, existing literature has not studied the EDT for cognitive transmission with imperfect periodic sensing.

3) Investigation of sensing error and transmission strategies for the secondary delay performance through an M/G/1 queuing formulation. 
The rest of this paper is organized as follows. In section II, we introduce the system model and problem formulation. In section III, we discuss the EDT analysis for work-preserving strategy with imperfect periodic sensing, non-work-preserving strategy with perfect periodic sensing, and non-work-preserving strategy with imperfect periodic sensing, in respective sub-sections. In section IV, Monte-Carlo simulation results are presented for secondary packet transmission in a general M/G/1 queuing set-up.

\section{System Model AND Problem Formulation}

We consider a cognitive transmission scenario where the SU opportunistically accesses a channel of the primary system for data transmission. The occupancy of that channel by the PU evolves independently according to a homogeneous continuous-time Markov chain with an average busy period of $\lambda$ and an average idle period of $\mu$. Thus, the duration of busy and idle periods are exponentially distributed ${ }^{1}$. The SU accesses the channel in an interweave fashion. Specifically, the SU can use the channel only after PU stops transmission. As soon as the PU restarts transmission, the SU instantaneously stops its transmission, and thus no interference is caused to the PU.

The SU monitors PU activity through spectrum sensing. Specific spectrum sensing methods, and the related problems, have been discussed in [37]-[42]. To ensure no interference to PU, we assume that during secondary transmission, the SU always continuously monitors PU activity. However, when not transmitting, it senses the channel periodically, with a period of $T_{s}$. In particular, if the PU is sensed busy, the SU will wait for $T_{s}$ time period and re-sense the channel. We consider two types of sensing scenarios. With perfect sensing, the SU will always be able to correctly sense the presence/absence of the PU. With imperfect sensing, there is a non-zero probability of false alarm, i.e. sensing a free channel to be busy, in each sensing attempt. The probability of false alarm is denoted by $p_{e}$. We assume that the chance of miss detection, i.e. sensing a busy channel to be free, is negligible ${ }^{2}$, which can be achieved in practice by adjusting the sensing thresholds properly. With the adapted sensing strategies, there is a small amount of time when the PU has stopped its transmission, but the SU has not yet acquired the channel,

\footnotetext{
${ }^{1}$ We assume a simplified traffic model (On-Off process) here, which is a very common used model in literature [33], [34]. Other more complex traffic models [35], [36] will be addressed in future work.

${ }^{2}$ The case with a non-zero miss detection probability will be considered in future work
} 


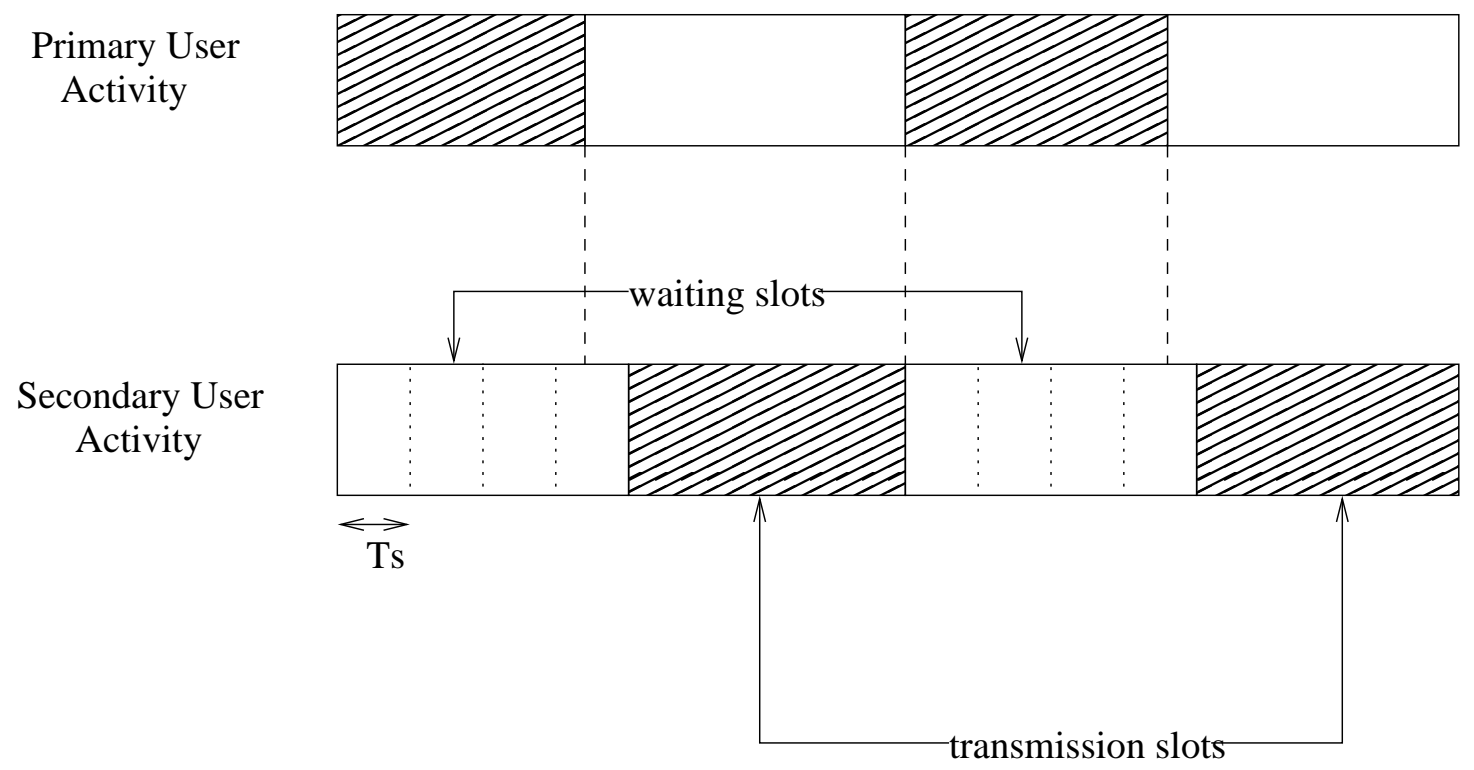

Fig. 1. Illustration of PU and SU activities and SU sensing - Perfect periodic sensing.

as illustrated in Fig. 1. Additional wait may occur due to false alarms, as shown in Fig. 2. The continuous period of time during which the PU is off and the SU is transmitting is referred to as a transmission slot. The continuous period of time during which SU is not transmitting is referred to as a waiting slot. A waiting slot contains the continuous period of time during which the PU is transmitting, or the PU has stopped transmission but the SU has not yet found the channel to be free.

In this work, we analyze the packet delivery time of secondary system. For work-preserving strategy, the packet delivery time duration contains an interleaved sequence of transmission time slots and waiting time slots. For non-work-preserving strategy, the packet delivery time duration includes an interleaved sequence of wasted transmission time slots and the waiting time slots, followed by a successful transmission time. Note that for non-work-preserving strategy, a transmission slot is wasted if its duration is less than the time required to transmit the packet. The resulting EDT for a packet is mathematically given by $T_{E D}=T_{w}+T_{t r}$, where $T_{w}$ is the total of the waiting time and wasted transmission times, if any, for the $\mathrm{SU}$, and $T_{t r}$ is the packet transmission time. In what follows, we derive the distribution of the EDT $T_{E D}$ for 


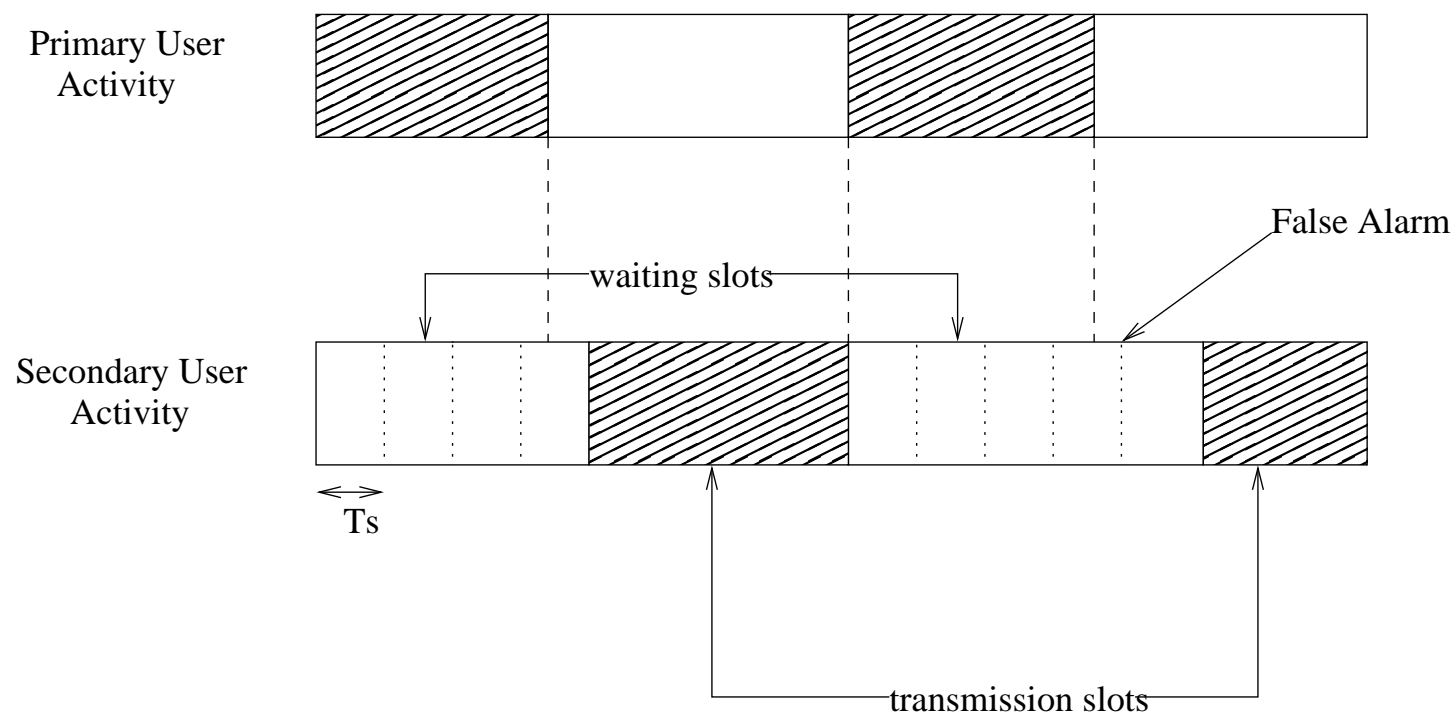

Fig. 2. Illustration of PU and SU activities and SU sensing - Imperfect periodic sensing.

work-preserving and non-work-preserving strategies, assuming a constant $T_{t r}{ }^{3}$.

${ }^{3}$ The transmission time, $T_{t r}$, in a general case, is a random variable depending on packet size and secondary channel condition when available, but can be considered a constant in many cases. As an example, for a fast-varying fading channel, $T_{t r}$ can be estimated as a constant depending on the ergodic channel capacity (Shannon capacity), given by $T_{t r} \approx \frac{H}{W \int_{0}^{\infty} \log _{2}(1+\gamma) f_{\gamma}(\gamma) d \gamma}$, where $H$ is the entropy of the packet, $W$ is the available bandwidth, and $f_{\gamma}(\gamma)$ is the PDF of the received SNR of the secondary channel. $T_{t r}$ will also be a constant if the channel is static. 


\section{EXTENDED DELIVERY TIME ANALysis}

In this section, we investigate the EDT of secondary system for a single packet arriving at a random point in time. This analysis applies to wireless sensor networks for health care monitoring, forest fire detection, air pollution monitoring, disaster prevention, landslide detection etc., where the transmitter needs to periodically transmit measurement data to the sink. The EDT essentially characterizes the data collection delay.

\section{A. Work-Preserving Strategy with Imperfect Periodic Sensing}

The EDT for SU packet transmission with work-preserving strategy consists of interleaved waiting slots and transmission slots. We assume, without loss of generality, that the packet arrives at $t=0$. Since SU senses the spectrum only at intervals of $T_{s}$, the distribution of the total wait time $T_{w}$ in this case is discrete, and will depend on whether the PU was on or off at $T=0$. The PDF of the EDT $T_{E D}$ for the SU can be calculated as

$$
\operatorname{Pr}\left[T_{E D}=n T_{s}+T_{t r}\right]=\frac{\lambda}{\lambda+\mu} \operatorname{Pr}\left[T_{w, p_{o n}}=n T_{s}\right]+\frac{\mu}{\lambda+\mu} \operatorname{Pr}\left[T_{w, p_{o f f}}=n T_{s}\right],
$$

where $\frac{\lambda}{\lambda+\mu}$ and $\frac{\mu}{\lambda+\mu}$ are the stationery probabilities that the PU is on and off at $t=0$, respectively, $\operatorname{Pr}\left[T_{w, p_{o n}}=n T_{s}\right]$ is the probability that the total SU waiting time is $n T_{s}$ when PU is on at $t=0$, and $\operatorname{Pr}\left[T_{w, p_{o f f}}=n T_{s}\right]$ the probability when PU is off at $t=0$.

The two probability mass functions (PMFs) $\operatorname{Pr}\left[T_{w, p_{o n}}=n T_{s}\right]$ and $\operatorname{Pr}\left[T_{w, p_{o f f}}=n T_{s}\right]$ above are calculated with the help of a discrete-time Markov chain as depicted in Fig. 3. State 1 corresponds to the case that PU is on when the channel is sensed. State 2 corresponds to the case that PU is off when the channel is sensed, but SU has failed to detect the free channel. State 3 represents the case that PU is off and SU has detected the free channel. This Markov chain differs from a conventional discrete-time Markov chain in the sense that it uses the concept of a variable time step, i.e. the period after which a possible transition takes place is not constant and depends on the current state. A time step after state 1 and state 2 is $T_{s}$ units, which represents the sensing interval. A time step after state 3 is random and exponentially distributed, which represents the SU transmission period. The transition probabilities of the Markov chain are calculated in terms of false alarm probability, the probability that the PU is on at a given sensing instant, provided that it was on at the previous sensing instant $T_{s}$ time units earlier, denoted by $\beta$, which is given 


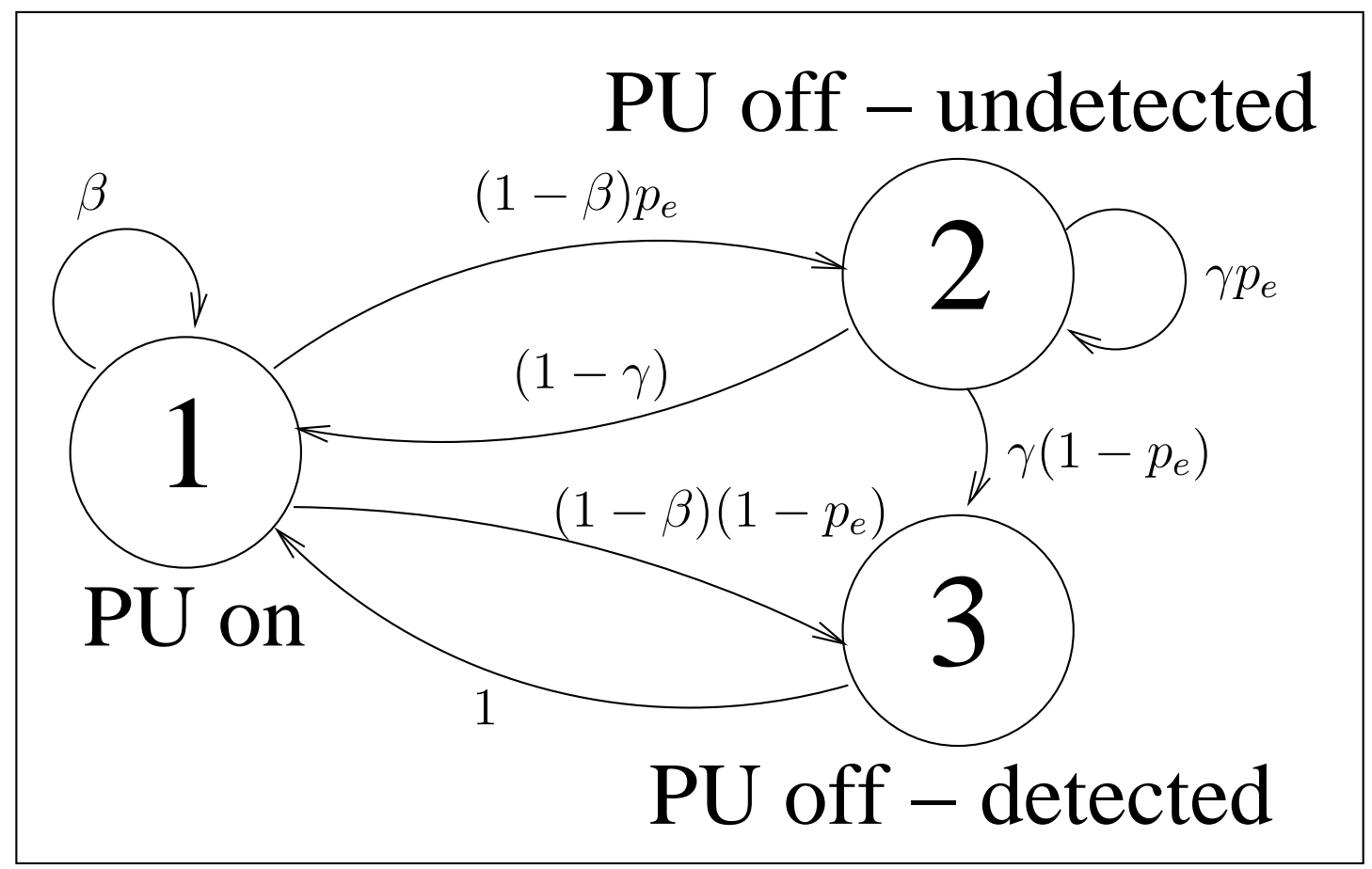

Fig. 3. State diagram for the cognitive system.

by [12]

$$
\beta=\frac{\lambda}{\lambda+\mu}+\frac{\mu}{\lambda+\mu} e^{-\left(\frac{1}{\lambda}+\frac{1}{\mu}\right) T_{s}} .
$$

and the probability that PU is off at a given sensing instant, provided that it was off at the previous sensing instant, denoted by $\gamma$, which can be calculated as

$$
\gamma=\frac{\mu}{\lambda+\mu}+\frac{\lambda}{\lambda+\mu} e^{-\left(\frac{1}{\lambda}+\frac{1}{\mu}\right) T_{s}} .
$$

Finally, the Markov chain will transit from state 3, after an exponentially distributed random duration, to state 1 with probability 1 , when PU starts transmission.

Based on the above model, the probability that the SU needs to wait $n$ sensing periods before transmitting exactly $k$ times for the case that PU is on at $t=0$, denoted by $\operatorname{Pr}\left[T_{w, p_{o n}}^{(k)}=n T_{s}\right]$, which is equal to the probability that the Markov chain visits state 3 exactly $k$ times after visiting 
states 1 and 2 exactly $n$ times, starting from state 1 at $t=0$, can be calculated as

$$
\begin{aligned}
\operatorname{Pr}\left[T_{w, P_{o n}}^{(k)}=\right. & \left.n T_{s}\right]=\sum_{\substack { i+j+2 \begin{subarray}{c}{i, j, m, z=n-k \\
i \leq k{ i + j + 2 \begin{subarray} { c } { i , j , m , z = n - k \\
i \leq k } } \\
{\substack{i \leq k \\
j}}\end{subarray}}\left[\gamma^{i+j}(1-\gamma)^{m} \beta^{z}(1-\beta)^{m+k} p_{e}^{i+j+m}\right. \\
& \left.\times\left(1-p_{e}\right)^{k} \cdot \frac{(m+k-1) !}{m ! i !(k-i) !} \cdot k \cdot\left(\begin{array}{c}
i+m+j-1 \\
j
\end{array}\right) \cdot\left(\begin{array}{c}
k+m+z-1 \\
z
\end{array}\right)\right],
\end{aligned}
$$

where the summation variable $i$ represents the number of transitions from state 2 to state $3, j$ the number of transitions from state 2 to state $2, m$ the number of transitions from state 2 to state 1 , and $z$ the number of transitions from state 1 to state 1 . Note that the number of transitions from state 1 to state 2 is $m+i$, while the number of transitions from state 1 to state 3 is $k-i$. Conditioning on the number of transmitting periods required for completing packet transmission, the PMF of SU wait time for $\mathrm{PU}$ on at $t=0, \operatorname{Pr}\left[T_{w, p_{o n}}=n T_{s}\right]$ can then be calculated as

$$
\operatorname{Pr}\left[T_{w, p_{o n}}=n T_{s}\right]=\sum_{k=1}^{\infty} \mathcal{P}_{k} \times \operatorname{Pr}\left[T_{w, p_{o n}}^{(k)}=n T_{s}\right]
$$

where $\mathcal{P}_{k}$ is the probability that the SU was successful in sending the packet in the $k^{\text {th }}$ transmission slot, given by [12]

$$
\mathcal{P}_{k}=\frac{T_{t r}^{k-1} e^{\frac{-T_{t r}}{\mu}}}{\mu^{k-1}(k-1) !} .
$$

In a similar manner, we define $\operatorname{Pr}\left[T_{w, p_{o f f}}^{(k)}=n T_{s}\right]$ as the probability that the time spent by the system in states 1 and 2 is $n T_{s}$ before it successfully finds the PU to be off exactly $k$ times, for the case when PU is off at $t=0$. Noting that if PU is off at $t=0$, there is a probability $\left(1-p_{e}\right)$ that the SU successfully senses PU to be off at $t=0$ (which becomes the first of the $k$ successful detections) and the Markov chain starts at state 3 , and a probability $p_{e}$ that the SU fails to sense the free channel, and the Markov chain starts at state $2 . \operatorname{Pr}\left[T_{w, p_{o f f}}^{(k)}=n T_{s}\right]$ can 
then be calculated as

$$
\begin{aligned}
& \operatorname{Pr}\left[T_{w, P_{o f f}}^{(k)}=n T_{s}\right]=p_{e} \\
& \times\left(\sum_{\substack{i+j+z-1=n-k \\
j, z \geq 0,1 \leq i \leq k}}\left[\gamma^{i+j} \beta^{z}(1-\beta)^{k-1} p_{e}^{i+j-1}\left(1-p_{e}\right)^{k} \times\left(\begin{array}{c}
k-1 \\
i-1
\end{array}\right) \cdot\left(\begin{array}{c}
i+j-1 \\
j
\end{array}\right) \cdot\left(\begin{array}{c}
k+z-2 \\
z
\end{array}\right)\right]\right. \\
& +\sum_{\substack{i+j+2 m+z-1=n-k \\
i, j, z \geq 0, m \geq 1}}\left[\gamma^{i+j}(1-\gamma)^{m} \beta^{z}(1-\beta)^{m+k-1} p_{e}^{i+j+m-1}\right. \\
& \left.\quad \times\left(1-p_{e}\right)^{k} \cdot \frac{(m+k-2) !}{m ! i !(k-i) !} \cdot(m k+i k-i) \cdot\left(\begin{array}{c}
i+m+j-1 \\
j
\end{array}\right) \cdot\left(\begin{array}{c}
k+m+z-2 \\
z
\end{array}\right)\right] \\
& +\left(1-p_{e}\right) \cdot \operatorname{Pr}\left[T_{w, k-1, P_{o n}}=n T_{s}\right]
\end{aligned}
$$

It follows that the PMF of SU wait time for PU off at $t=0$ case, $\operatorname{Pr}\left[T_{w, p_{\text {off }}}=n T_{s}\right]$ can be calculated similar to $\operatorname{Pr}\left[T_{w, p_{o n}}=n T_{s}\right]$ in Eq. (5), but using $\operatorname{Pr}\left[T_{w, p_{o f f}}^{(k)}=n T_{s}\right]$ in Eq. (7) in place of $\operatorname{Pr}\left[T_{w, p_{o n}}^{(k)}=n T_{s}\right]$.

Fig. 4 plots the PMF of the EDT with work-preserving strategy, $\operatorname{Pr}\left[T_{E D}=n T_{s}+T_{t r}\right]$. The corresponding plot for simulation results is also shown. The perfect match between analytical and simulation results verifies our analytical approach.

\section{B. Non-Work-Preserving Strategy with Perfect Periodic Sensing}

For non-work-preserving strategy, the EDT for packet transmission by the SU consists of interleaved waiting slots and wasted transmission slots, followed by the final successful transmission slot of duration $T_{t r}$. The distribution of waiting time $T_{w}$ depends on whether the PU was on or off at the instant of packet arrival. We denote the PDF of the waiting time of the SU with perfect periodic sensing for the case when PU is on at the instant of packet arrival, and for the case when PU is off at that instant, by $f_{T_{w}, p_{o n}}^{(p)}(t)$ and $f_{T_{w}, p_{o f f}}^{(p)}(t)$, respectively. The PDF of the EDT $T_{E D}$ for the $\mathrm{SU}$ is then given by

$$
f_{T_{E D}(p)}^{(p)}(t)=\frac{\lambda}{\lambda+\mu} f_{T_{w}, p_{o n}}^{(p)}\left(t-T_{t r}\right)+\frac{\mu}{\lambda+\mu} f_{T_{w}, p_{o f f}}^{(p)}\left(t-T_{t r}\right) .
$$

The two probability density functions $f_{T_{w}, p_{o n}}(t)$ and $f_{T_{w}, p_{o f f}}(t)$ above are calculated independently as follows. 


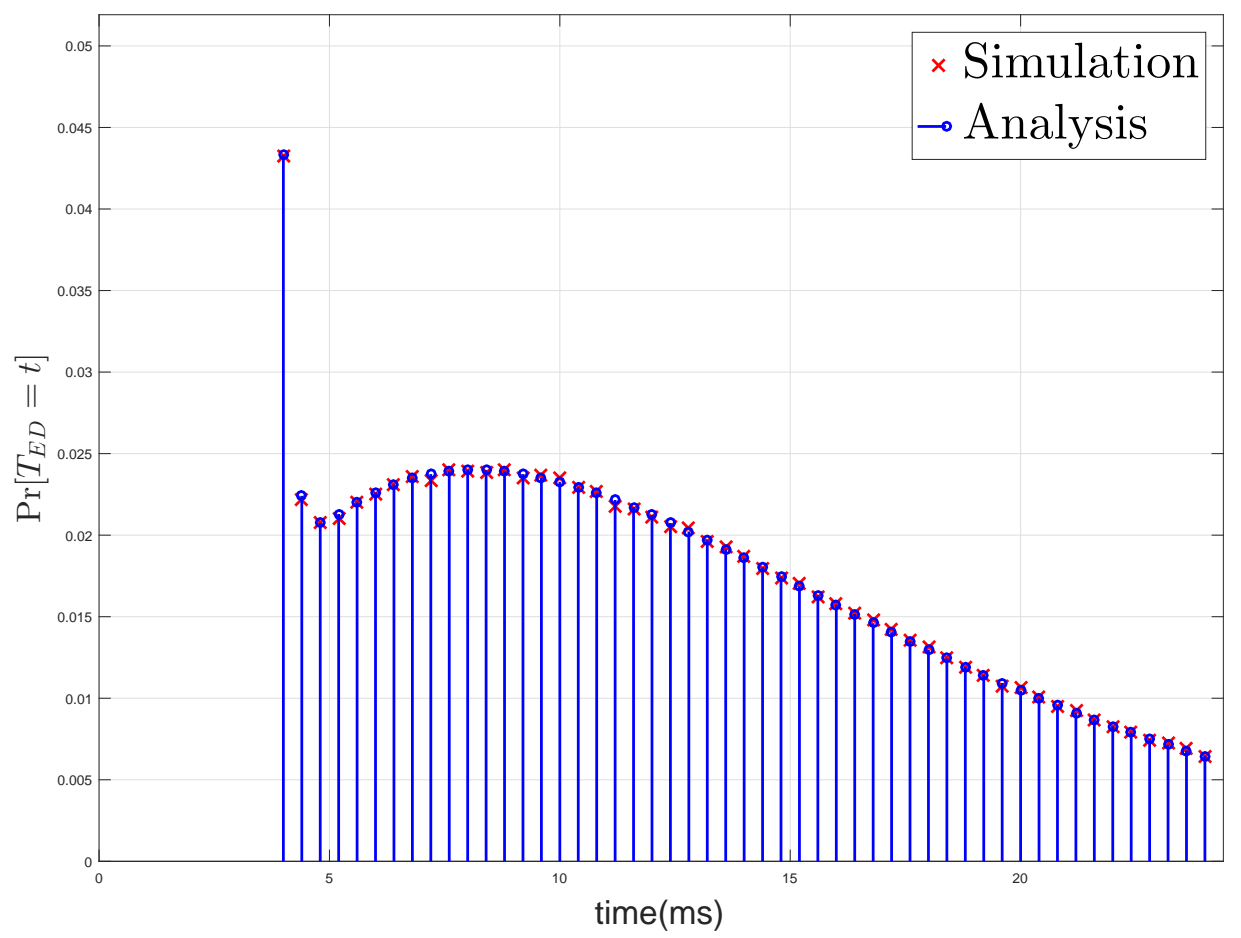

Fig. 4. Simulation verification for the PMF of $T_{E D}$ with work-preserving strategy, imperfect periodic sensing $\left(T_{t r}=4 \mathrm{~ms}\right.$, $\lambda=3 \mathrm{~ms}, \mu=2 \mathrm{~ms}, T_{s}=0.4 \mathrm{~ms}$, and $\left.p_{e}=0.2\right)$.

Let $\widehat{\mathcal{P}}_{k}$ be the probability that the SU was successful in sending the packet in the $k^{\text {th }}$ transmission slot with non-work-preserving strategy. This means that each of the first $(k-1)$ slots had a time duration of less than $T_{t r}$, while the $k^{\text {th }}$ transmission slot had a duration more than $T_{t r}$. Thus, $\widehat{\mathcal{P}}_{k}$ can be calculated, while noting that the duration of secondary transmission slots is exponentially distributed with mean $\mu$, as

$$
\widehat{\mathcal{P}}_{k}=e^{-\frac{T_{t r}}{\mu}} \cdot\left(1-e^{-\frac{T_{t r}}{\mu}}\right)^{k-1} .
$$

For the case when PU is off at the instant of packet arrival, if a certain packet is transmitted completely in the $k^{\text {th }}$ transmission slot, then the total wait time for that packet includes $(k-1)$ secondary waiting slots and $(k-1)$ wasted transmission slots. Note that the duration of each of these $(k-1)$ waiting slots, denoted by the discrete random variable $T_{\text {wait }}$, which is equal to PU on time, consists of multiple $T_{s}$, and follows a geometric distribution with PDF given by $\operatorname{Pr}\left[T_{\text {wait }}=n T_{s}\right]=(1-\beta) \beta^{n-1}$, where $\beta$ is given by Eq. (2), which is a constant due to 


$$
\begin{aligned}
& f_{T_{w}, p_{o f f}}^{(p)}(t)=e^{-\frac{T_{t r}}{\mu}} \delta(t)+\sum_{n=1}^{\infty}\left[\frac{(1-\beta) \beta^{n-1}}{\mu} e^{-\frac{\left(t-n T_{s}\right)}{\mu}} e^{-\frac{T_{t r}}{\mu}}{ }_{1} F_{1}\left(1-n ; 1 ;-\frac{1-\beta}{\beta} \frac{t-n T_{s}}{\mu}\right)\right. \\
& \left.+\sum_{i=1}^{n}\left[(-1)^{i} e^{-(i+1) \frac{T_{t r}}{\mu}}\left(\begin{array}{c}
n-1 \\
i-1
\end{array}\right) \frac{1}{(i-1) !} \frac{\left(t-i T_{t r}-n T_{s}\right)^{i-1}}{\mu^{i}}(1-\beta)^{i} \beta^{n-i} e^{\frac{-\left(t-n T_{s}-i T_{t r}\right)}{\mu}} \times{ }_{2} F_{2}\left(i+1, i-n ; i, i ;-\frac{1-\beta}{\beta} \frac{\left(t-n T_{s}-i T_{t r}\right)}{\mu}\right)\right]\right] . \\
& \begin{array}{l}
f_{T_{w}, p_{\text {on }}}^{(p)}(t)=e^{-\frac{T_{t r}}{\mu}} \sum_{n=2}^{\infty}(n-1) \frac{(1-\beta)^{2} \beta^{n-2}}{\mu} e^{-\frac{t-n T_{s}}{\mu}}{ }_{1} F_{1}\left(2-n ; 2 ;-\frac{1-\beta}{\beta} \cdot \frac{t-n T_{s}}{\mu}\right)+e^{-\frac{T_{t r}}{\mu}} \sum_{n=1}^{\infty}(1-\beta) \beta^{n-1} \delta\left(t-n T_{s}\right) \\
+\sum_{n=1}^{\infty} \sum_{i=1}^{n-1}\left[(-1)^{i} e^{-(i+1) \frac{T_{t r}}{\mu}}\left(\begin{array}{c}
n-1 \\
i
\end{array}\right)(1-\beta)^{i+1} \beta^{n-i-1} \times \frac{t^{i-1} e^{-\frac{t-n T_{s}-i T_{t r}}{\mu}}}{(i-1) ! \mu^{i}}{ }_{1} F_{1}\left(i+1-n ; i ;-\frac{1-\beta}{\beta} \cdot \frac{t-n T_{s}-i T_{t r}}{\mu}\right)\right] .
\end{array}
\end{aligned}
$$

the memoryless property of exponential distribution, while the duration of each of the previous $(k-1)$ wasted secondary transmission slots, denoted by the random variable $T_{\text {waste }}$, follows a truncated exponential distribution, with PDF given by

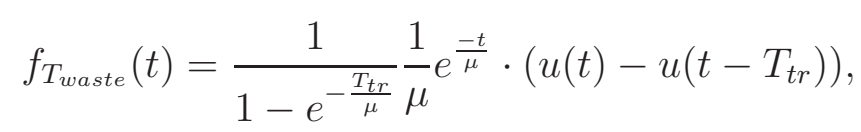

where $u(t)$ is the unit step function. The moment generating function (MGF) of $T_{w, p_{o f f}}$ for the perfect periodic sensing case, $\mathcal{M}_{T_{w}, p_{o f f}}^{(p)}(s)$, can be calculated as

$$
\mathcal{M}_{T_{w}, p_{\text {off }}}^{(p)}(s)=\sum_{k=1}^{\infty} \widehat{\mathcal{P}}_{k} \cdot\left(\mathcal{M}_{T_{\text {wait }}}^{(p)}(s)\right)^{k-1} \cdot\left(\mathcal{M}_{T_{\text {waste }}}(s)\right)^{k-1}
$$

where $\mathcal{M}_{T_{\text {wait }}}^{(p)}(s)$ is the MGF of $T_{\text {wait }}$, given by

$$
\mathcal{M}_{T_{\text {wait }}}^{(p)}(s)=\sum_{n=1}^{\infty}(1-\beta) \beta^{n-1} e^{n s T_{s}},
$$

and $\mathcal{M}_{T_{\text {waste }}}(s)$ is the MGF of $T_{\text {waste }}$, given by

$$
\mathcal{M}_{T_{\text {waste }}}(s)=\frac{1-e^{T_{t r}\left(s-\frac{1}{\mu}\right)}}{(1-\mu s)\left(1-e^{-\frac{T_{t r}}{\mu}}\right)} .
$$

Substituting Eqs. (9), (13), and (12) into Eq. (11), performing some manipulation, and taking the inverse MGF, we obtain the expression for $f_{T_{w}, p_{o f f}}^{(p)}(t)$ as shown in Eq. (14), where ${ }_{1} F_{1}(., .,$. is the generalized Hypergeometric function [43].

For the case when PU is on at the instant of packet arrival, the PDF of $T_{w, p_{o n}}$ for the periodic sensing case can be similarly calculated as shown in Eq. (15). Note that the sequence of impulses corresponds to the case that the packet is transmitted in the first transmission attempt on acquiring the channel after a random number of sensing intervals/attempts. 


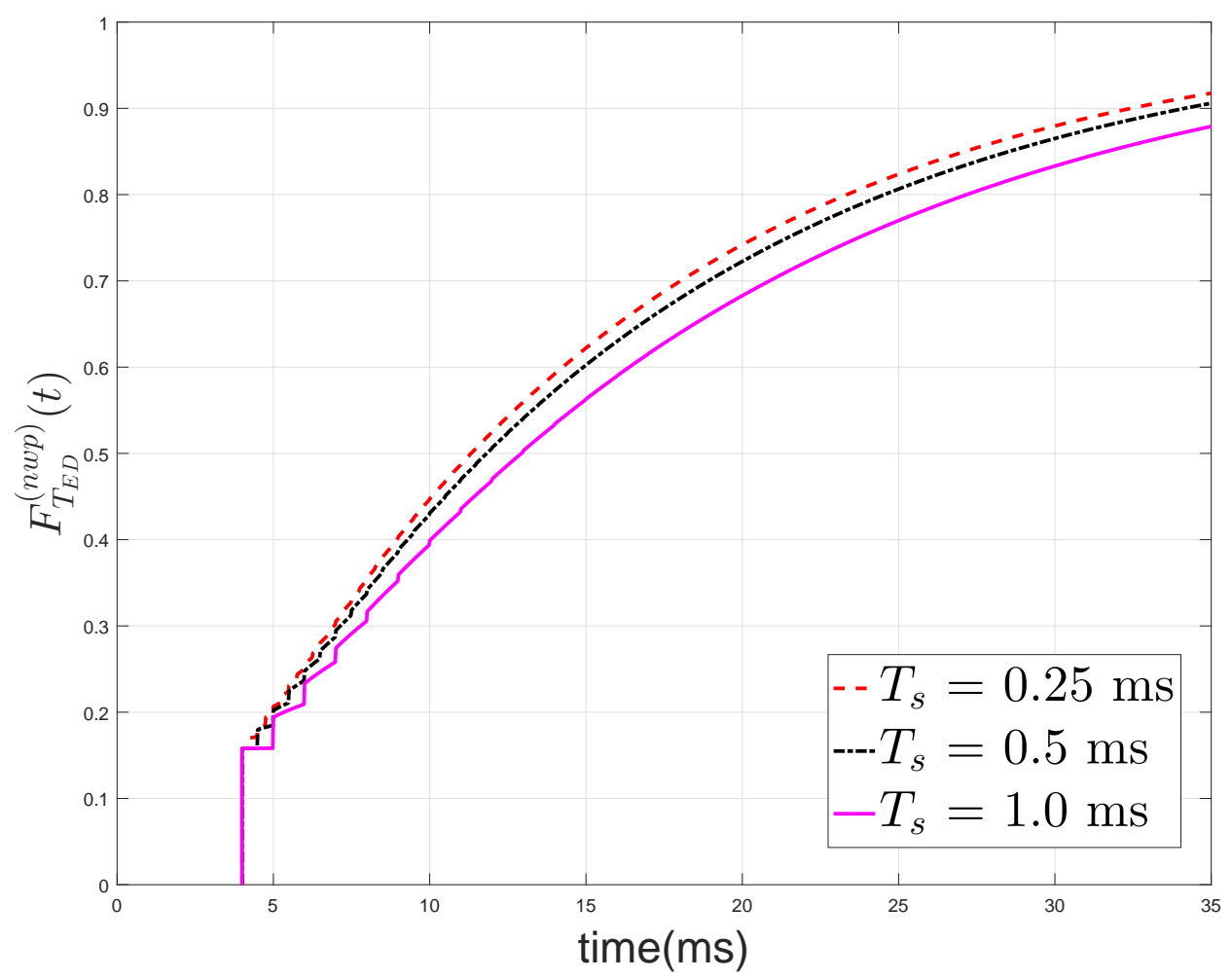

Fig. 5. Analytical CDF of $T_{E D}$ with non-work-preserving strategy, perfect periodic sensing $\left(T_{t r}=4 \mathrm{~ms}, \lambda=3 \mathrm{~ms}\right.$, and $\mu=2$ $\mathrm{ms})$.

Fig. 5 plots the cumulative distribution function (CDF) of the EDT for non-work-preserving strategy with periodic sensing, $F_{T_{E D}}^{(p)}(t)$, obtained by numerical integration of the analytical PDF expression given by Eq. (8), for various values of period $T_{s}$. As expected, longer $T_{s}$ leads to slower increase in the CDF values.

\section{Non-Work-Preserving Strategy with Imperfect Periodic Sensing}

We now consider the effect of imperfect sensing on non-work-preserving strategy. Following similar reasoning in previous subsection, the MGF of the total wait time with non-workpreserving strategy, $\mathcal{M}_{T_{w}}^{(n w p)}(s)$ can be defined as

$$
\mathcal{M}_{T_{w}}^{(n w p)}(s)=\sum_{k=1}^{\infty} \widehat{\mathcal{P}}_{k} \times \mathcal{M}_{T_{w}}^{(k)}(s) \times\left(\mathcal{M}_{T_{\text {wast }}}(s)\right)^{k-1}
$$


where $\widehat{\mathcal{P}}_{k}$ is the probability that the packet transmission was successful only on the $k^{\text {th }}$ transmision slot, given in Eq. (9), $\mathcal{M}_{T_{\text {waste }}}(s)$ is the MGF of the time duration of a wasted transmission slot, given by Eq. (13), and $\mathcal{M}_{T_{w}}^{(k)}(s)$ is the MGF of the time duration of $k$ waiting slots for a packet arriving at a random point in time, given by

$$
\mathcal{M}_{T_{w}}^{(k)}(s)=\sum_{n=0}^{\infty} \operatorname{Pr}\left[T_{w}^{(k)}=n T_{s}\right] e^{n s T_{s}},
$$

where

$$
\operatorname{Pr}\left[T_{w}^{(k)}=n T_{s}\right]=\frac{\lambda}{\lambda+\mu} \operatorname{Pr}\left[T_{w, P_{o n}}^{(k)}=n T_{s}\right]+\frac{\mu}{\lambda+\mu} \operatorname{Pr}\left[T_{w, P_{o f f}}^{(k)}=n T_{s}\right],
$$

with $\operatorname{Pr}\left[T_{w, p_{o n}}^{(k)}=n T_{s}\right]$ and $\operatorname{Pr}\left[T_{w, p_{o f f}}^{(k)}=n T_{s}\right]$ given in Eqs. (4) and (7), respectively. Substituting Eqs. (9), (13), and (17) into Eq. (16), performing some manipulation, and taking the inverse MGF, we obtain the PDF of EDT for non-work-preserving strategy with imperfect periodic sensing, while noting $T_{E D}=T_{w}+T_{t r}$, as

$$
\begin{aligned}
& f_{T_{E D}}^{(n w p)}(t)=\sum_{n=0}^{\infty} \sum_{k=2}^{n+1} \sum_{i=0}^{k-1} \operatorname{Pr}\left[T_{w}^{(k)}=n T_{s}\right]\left(\begin{array}{c}
k-1 \\
i
\end{array}\right) \cdot(-1)^{i} \\
& \times e^{-(i+1) \frac{T_{t r}}{\mu}} \frac{\left[t-n T_{s}-(i+1) T_{t r}\right]^{k-2}}{\Gamma[k-1] \mu^{k-1}} e^{-\frac{\left[t-n T_{s}-(i+1) T_{t r}\right]}{\mu}} \\
& +\sum_{n=0}^{\infty} \operatorname{Pr}\left[T_{w}^{(k)}=n T_{s}\right] e^{-\frac{T_{t r}}{\mu}} \delta\left[t-n T_{s}-T_{t r}\right] .
\end{aligned}
$$

Fig. 6 plots the cumulative distribution function (CDF) of the EDT with work-preserving strategy, $F_{T_{E D}}^{(n w p)}(t)$, obtained by numerical integration of the analytical PDF expression given by Eq. (19), for various values of $p_{e}$. The plot for $p_{e}=0$ represents the case with perfect periodic sensing. The corresponding plots for the simulation results are also shown. It is evident from the plots that increasing the value of $p_{e}$ leads to longer EDT. The perfect match between analytical and simulation results verifies our analytical approach.

Fig. 7 compares the average EDT for two different values of $T_{t r}$, for various values of false alarm probability, $p_{e}$. As expected, an increase in the value of $p_{e}$ increases the average EDT. The average EDT for non-work-preserving strategy is always smaller than that for work-preserving strategy, with the difference diminishing for a small value of $T_{t r}$. 


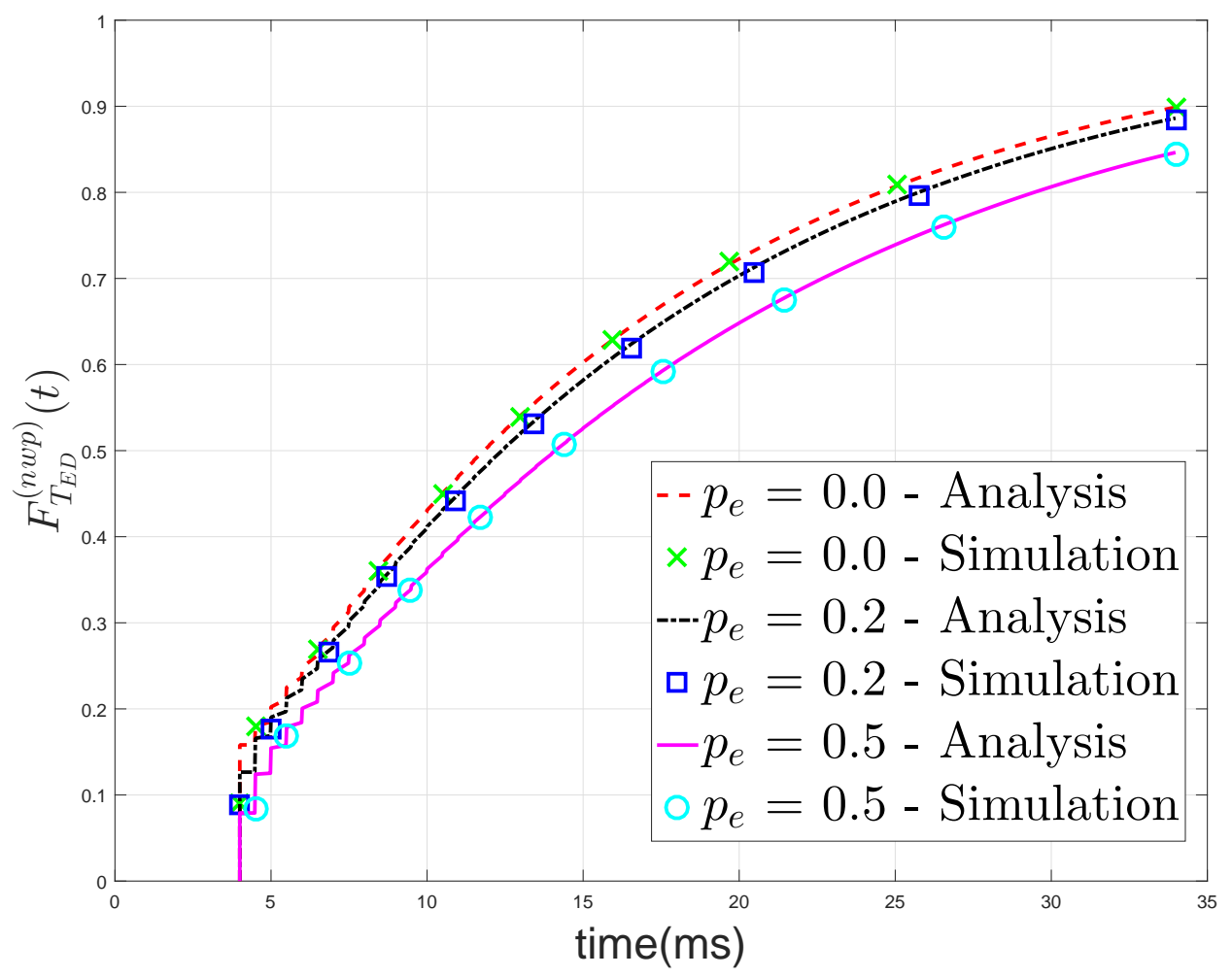

Fig. 6. Simulation verification for the CDF of $T_{E D}$ with non-work-preserving strategy, imperfect periodic sensing $\left(T_{t r}=4\right.$ $\mathrm{ms}, \lambda=2 \mathrm{~ms}, \mu=3 \mathrm{~ms}$, and $T_{s}=0.5 \mathrm{~ms}$ ). 


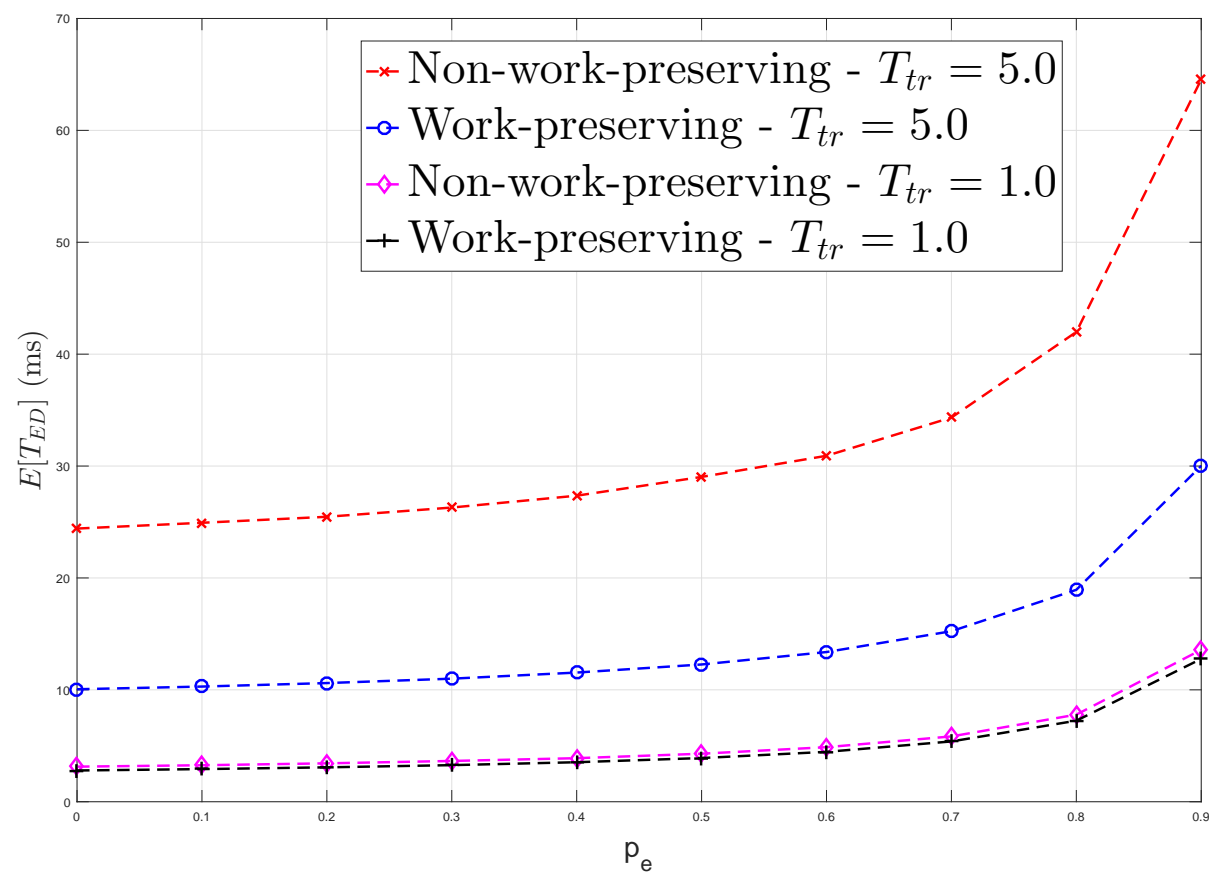

Fig. 7. Comparison of average EDT for work-preserving and non-work-preserving strategies with imperfect periodic sensing $\left(\lambda=2 \mathrm{~ms}, \mu=3 \mathrm{~ms}\right.$, and $\left.T_{s}=0.5 \mathrm{~ms}\right)$. 


\section{Queuing RESUlts}

In this section, we consider the average transmission delay for the secondary system in a queuing set-up, as an application of the analytical results in previous section. In particular, the secondary traffic intensity is high and, as such, a first-in-first-out queue is introduced to hold packets until transmission. We assume that equal-sized packet arrival follows a Poisson process with intensity $\frac{1}{\psi}$ [12]. For the sake of clarity, transmission time $T_{t r}$ of all packets is assumed to be a fixed constant. As such, the secondary packet transmission can be modelled as a general $\mathrm{M} / \mathrm{G} / 1$ queue, where the service time is closely related to the EDT studied in the previous section. Following the similar approach in Sec. IV-B of [12], the queuing delay for different scenarios discussed in section III can be analyzed. We focus on the perfect periodic sensing case with non-work-preserving strategy in the following, while noting the analysis for the other scenarios can be similarly solved. Note the service time of a packet depends on whether the PU is on or off when the packet is available for transmission.

\section{A. Moments of Service Time}

To facilitate queuing analysis, we now calculate the first and second moments of the service time for these two types of packets.

First moments. The service time for packets seeing PU off is denoted by $S T_{p_{\text {off }}}$. Noting that $S T_{p_{o f f}}=T_{w, p_{o f f}}+T_{t r}$, due to the memoryless property of the non-work-preserving strategy, we can calculate its first moment $E\left[S T_{p_{\text {off }}}\right]$ by following the total expectation theorem as

$$
E\left[S T_{p_{o f f}}\right]=e^{-\frac{T_{t r}}{\mu}} \cdot T_{t r}+\left(1-e^{-\frac{T_{t r}}{\mu}}\right) \times E\left[\left(T_{\text {waste }}+T_{\text {wait }}+S T_{p_{o f f}}\right)\right] .
$$

Here the first addition term corresponds to the case that the complete packet is successfully transmitted in the first transmission slot, and the second addition term refers to the case when the complete packet is not successfully transmitted. For periodic sensing with non-work-preserving strategy, it can be shown, from Eqs. (12) and (13), that $E\left[T_{\text {wait }}\right]=\frac{T_{s}}{1-\beta}$ and $E\left[T_{\text {waste }}\right]=\mu-$ $T_{t r} \frac{e^{-\frac{T_{t r}}{\mu}}}{1-e^{-\frac{T_{t r}}{\mu}}}$. The first moment of $S T_{p_{o f f}}$ can be calculated from Eq. (20) as

$$
E\left[S T_{p_{o f f}}\right]=\left(e^{\frac{T_{t r}}{\mu}}-1\right)\left(\mu+\frac{T_{s}}{1-\beta}\right)
$$


Since the case with PU on at the instant of packet arrival is precisely the same as the case of PU off at the instant of packet arrival preceded by a waiting slot, we can calculate $E\left[S T_{p_{o n}}\right]$, the first moment of the service time for packets seeing PU on, as

$$
E\left[S T_{p_{o n}}\right]=E\left[S T_{p_{o f f}}\right]+\frac{T_{s}}{1-\beta}=\left(e^{\frac{T_{t r}}{\mu}}-1\right) \cdot \mu+e^{\frac{T_{t r}}{\mu}} \cdot \frac{T_{s}}{1-\beta} .
$$

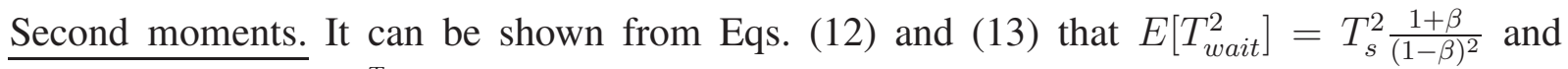
$E\left[T_{\text {waste }}^{2}\right]=2 \mu^{2}+\frac{e^{-\frac{T_{t r}}{\mu}}}{1-e^{-\frac{T_{t r}}{\mu}}}\left(-T_{t r}^{2}-2 \mu T_{t r}\right)$. Using a similar equation as Eq. (20) for second moment, and simplifying, we obtain

$$
\begin{aligned}
& E\left[S T_{p_{o f f}}^{2}\right]=e^{\frac{T_{t r}}{\mu}}\left[-2 T_{t r} \frac{T_{s}}{1-\beta}-2 \mu T_{t r}\right] \\
& +\left(e^{\frac{T_{t r}}{\mu}}-1\right)\left(e^{\frac{T_{t r}}{\mu}}\left[2 \mu \frac{T_{s}}{1-\beta}+2 \mu^{2}\right]+T_{s}^{2} \frac{1+\beta}{(1-\beta)^{2}}\right)+\left(e^{\frac{T_{t r}}{\mu}}-1\right)^{2}\left[2 \frac{T_{s}^{2}}{(1-\beta)^{2}}+2 \mu \frac{T_{s}}{1-\beta}\right] .
\end{aligned}
$$

Similarly, the second moment of the service time for packets seeing PU on can be defined as

$$
\begin{aligned}
E\left[S T_{p_{o n}}^{2}\right] & =E\left[\left(T_{\text {wait }}+S T_{p_{o f f}}\right)^{2}\right], \text { and calculated as } \\
E\left[S T_{p_{o n}}^{2}\right] & =e^{\frac{T_{t r}}{\mu}}\left[-2 T_{t r} \frac{T_{s}}{1-\beta}-2 \mu T_{t r}+T_{s}^{2} \frac{1+\beta}{(1-\beta)^{2}}\right]+2\left(e^{\frac{T_{t r}}{\mu}}-1\right) e^{\frac{T_{t r}}{\mu}}\left(\mu+\frac{T_{s}}{1-\beta}\right)^{2} .
\end{aligned}
$$

Finally, these moments can be substituted into Eqs. (45)-(55) of [12] to calculate the average queuing delay.

\section{B. Numerical Results}

Here we present selected numerical results for the average queuing delay for secondary packets in a queuing set-up as described above.

Fig. 8 plots the average queuing delay of secondary packets against the packet arrival rate, for various values of false alarm probability $p_{e}$, for work-preserving strategy. As expected, the average delay is always greater for a larger value of the false alarm probability. Similar observation can be made for non-work-preserving case.

Fig. 9 shows the average queuing delay against the rate of data packet arrival, for various values of $T_{t r}$, for work-preserving and non-work-preserving strategies with imperfect periodic sensing. It can be seen that as expected, work-preserving strategy always performs better than non-workpreserving strategy. Also, the performance difference between the two strategies reduces as the packet transmission time $T_{t r}$ decreases, as shown by the vertical lines in the figure. 


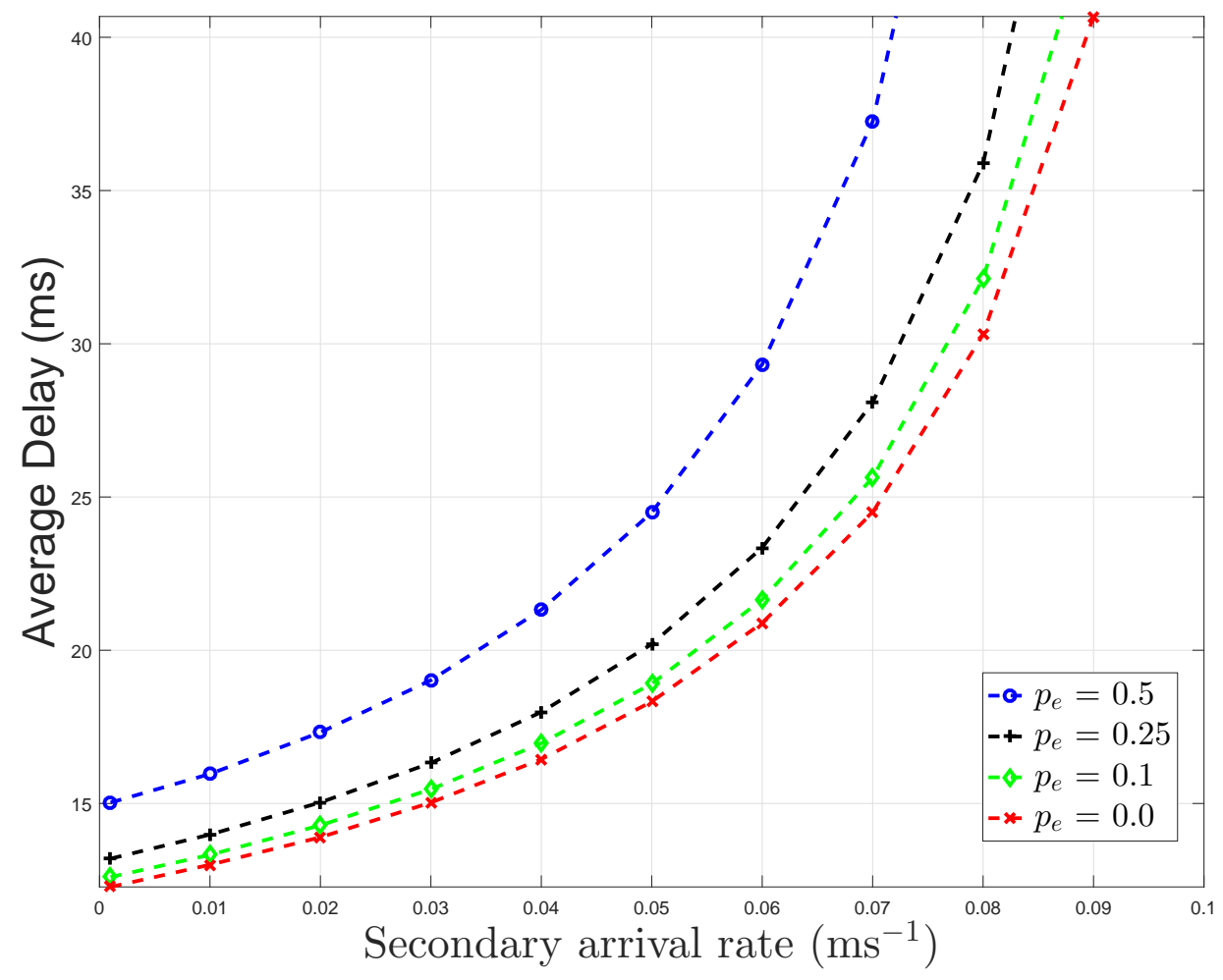

Fig. 8. Average queuing delay for imperfect periodic sensing with work-preserving strategy $\left(T_{s}=0.5 \mathrm{~ms}, \lambda=5 \mathrm{~ms}, \mu=3\right.$ $\mathrm{ms}$, and $T=3 \mathrm{~ms}$ ) 


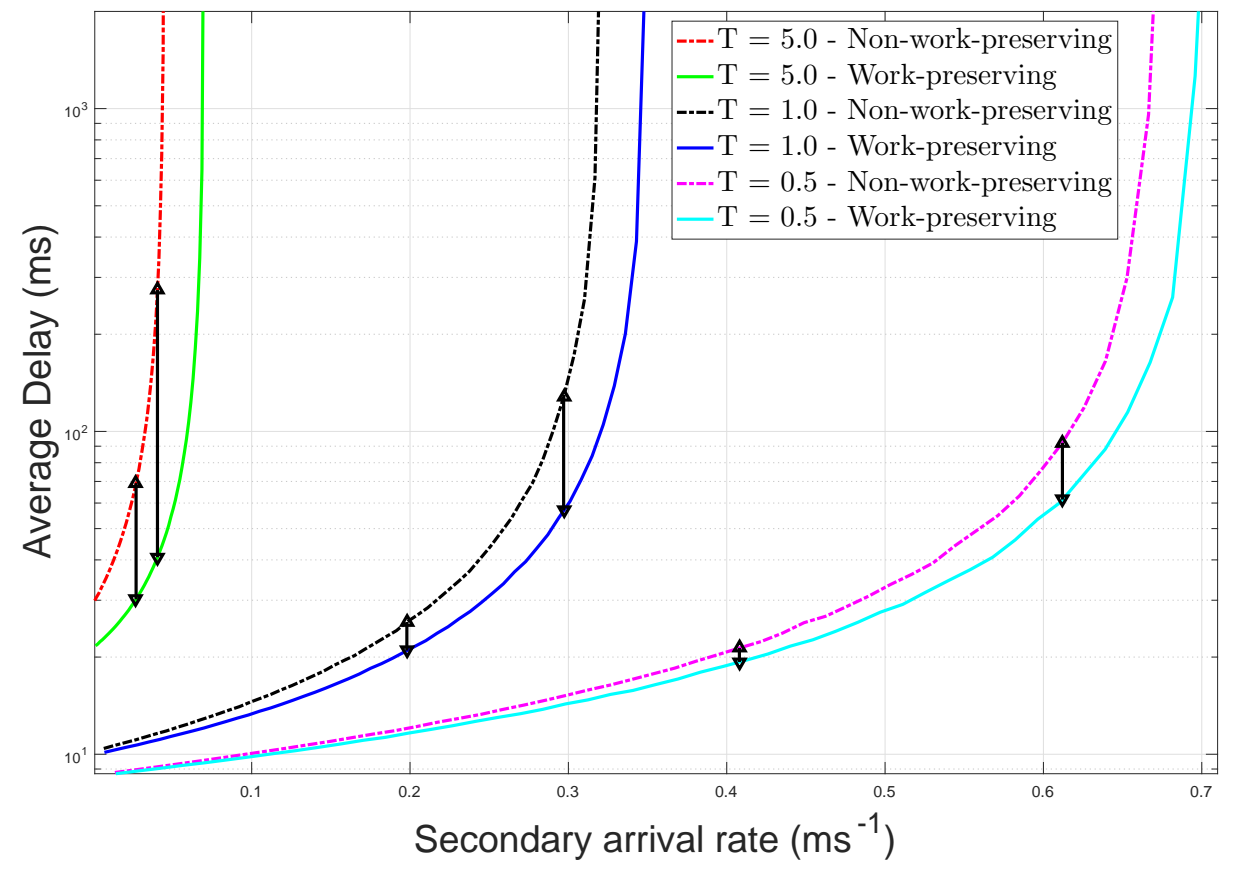

Fig. 9. Average queuing delay with imperfect periodic sensing $\left(T_{s}=0.5 \mathrm{~ms}, \lambda=10 \mathrm{~ms}, \mu=6 \mathrm{~ms}, p_{e}=0.25\right)$ 


\section{CONCLUSION}

This paper presented new results on the extended delivery time (EDT) of a data packet appearing at the secondary user in an interweave cognitive setup. Exact analytical results for the probability distribution of the EDT for a fixed-size data packet were obtained. We also analyzed the effect of imperfect periodic sensing for both work-preserving and non-preserving strategies. Simulation results were presented to verify the analytical results. These analytical results will facilitate the design and optimization of secondary systems for diverse target applications.

\section{REFERENCES}

[1] S. Haykin, "Cognitive radio: brain-empowered wireless communications," IEEE J. Sel. Areas Commun., vol. 23, pp. 201220, Feb 2005.

[2] J. Mitola and J. Maguire, G.Q., “Cognitive radio: making software radios more personal," IEEE Pers. Commun., vol. 6, pp. 13-18, Aug 1999.

[3] R. Thomas, L. DaSilva, and A. MacKenzie, "Cognitive networks," in 1st IEEE Int. Symp. on New Frontiers in Dynamic Spectrum Access Netw., 2005. DySPAN 2005. 2005, pp. 352-360, Nov 2005.

[4] I. F. Akyildiz, W.-Y. Lee, M. C. Vuran, and S. Mohanty, "Next generation/dynamic spectrum access/cognitive radio wireless networks: A survey," Comput. Netw. J., vol. 50, no. 13, pp. 2127 - 2159, 2006.

[5] M. Islam, C. Koh, S. W. Oh, X. Qing, Y. Lai, C. Wang, Y.-C. Liang, B. Toh, F. Chin, G. Tan, and W. Toh, "Spectrum survey in Singapore: Occupancy measurements and analyses," in Proc. 3rd Int. Conf. Cognitive Radio Oriented Wireless Netw. and Commun., 2008. CrownCom 2008., pp. 1-7, May 2008.

[6] B. Hamdaoui, "Adaptive spectrum assessment for opportunistic access in cognitive radio networks," IEEE Trans. Wireless Commun., vol. 8, pp. 922-930, Feb 2009.

[7] Q. Zhao, S. Geirhofer, L. Tong, and B. Sadler, “Opportunistic spectrum access via periodic channel sensing," IEEE Trans. Signal Process., vol. 56, pp. 785-796, Feb 2008.

[8] Q. Zhao, L. Tong, A. Swami, and Y. Chen, "Decentralized cognitive MAC for opportunistic spectrum access in ad hoc networks: A pomdp framework," IEEE J. Sel. Areas Commun., vol. 25, pp. 589-600, April 2007.

[9] A. Goldsmith, S. Jafar, I. Maric, and S. Srinivasa, "Breaking spectrum gridlock with cognitive radios: An information theoretic perspective," Proc. of the IEEE, vol. 97, pp. 894-914, May 2009.

[10] F. Borgonovo, M. Cesana, and L. Fratta, "Throughput and delay bounds for cognitive transmissions," in Advances in Ad Hoc Networking (P. Cuenca, C. Guerrero, R. Puigjaner, and B. Serra, eds.), vol. 265 of IFIP International Federation for Information Processing, pp. 179-190, Springer US, 2008.

[11] C.-W. Wang and L.-C. Wang, "Analysis of reactive spectrum handoff in cognitive radio networks," IEEE J. Sel. Areas Commun., vol. 30, pp. 2016-2028, November 2012.

[12] M. Usman, H.-C. Yang, and M.-S. Alouini, "Extended delivery time analysis for cognitive packet transmission with application to secondary queuing analysis," IEEE Trans. Wireless Commun., vol. 14, pp. 5300-5312, Oct 2015.

[13] F. Khan, K. Tourki, M.-S. Alouini, and K. Qaraqe, "Delay performance of a broadcast spectrum sharing network in Nakagami-m fading," IEEE Trans. Veh. Technol., vol. 63, pp. 1350-1364, March 2014. 
[14] L. Sibomana, H.-J. Zepernick, H. Tran, and C. Kabiri, "Packet transmission time for cognitive radio networks considering interference from primary user," in 9th Int. Wireless Commun. and Mobile Computing Conf. (IWCMC), 2013, pp. 791-796, July 2013.

[15] H. Tran, T. Duong, and H.-J. Zepernick, "Delay performance of cognitive radio networks for point-to-point and point-tomultipoint communications," EURASIP J. on Wireless Commun. and Networking, vol. 2012, no. 1, pp. 1-15, 2012.

[16] A. Farraj, S. Miller, and K. Qaraqe, "Queue performance measures for cognitive radios in spectrum sharing systems," in IEEE GLOBECOM Workshops (GC Wkshps), 2011, pp. 997-1001, Dec 2011.

[17] F. Gaaloul, H.-C. Yang, R. Radaydeh, and M.-S. Alouini, "Switch based opportunistic spectrum access for general primary user traffic model," IEEE Wireless Commun. Lett., vol. 1, pp. 424-427, October 2012.

[18] Z. Liang and D. Zhao, "Quality of service performance of a cognitive radio sensor network," in Proc. IEEE Int. Conf. Commun. (ICC), 2010, pp. 1-5, May 2010.

[19] Z. Liang, S. Feng, D. Zhao, and X. Shen, "Delay performance analysis for supporting real-time traffic in a cognitive radio sensor network," IEEE Trans. Wireless Commun., vol. 10, pp. 325-335, January 2011.

[20] S. Kandeepan, C. Saradhi, M. Filo, and R. Piesiewicz, "Delay analysis of cooperative communication with opportunistic relay access," in Proc. IEEE 73rd Veh. Technol. Conf. (VTC Spring), 2011, pp. 1-5, May 2011.

[21] H. Li and Z. Han, "Queuing analysis of dynamic spectrum access subject to interruptions from primary users," in 5th Int. Conf. Cognitive Radio Oriented Wireless Netw. Commun. (CROWNCOM), 2010 Proc., pp. 1-5, June 2010.

[22] M. Kahvand, M. Soleimani, and M. Dabiranzohouri, "Channel selection in cognitive radio networks: A new dynamic approach,” in Communications (MICC), 2013 IEEE Malaysia International Conference on, pp. 407-411, Nov 2013.

[23] A. Namanya and J. Pagna-Disso, "Performance modelling and analysis of the delay aware routing metric in cognitive radio ad hoc networks," in Wireless and Mobile Networking Conference (WMNC), 2013 6th Joint IFIP, pp. 1-8, April 2013.

[24] Q. Liu, X. Wang, and Y. Cui, "Robust and adaptive scheduling of sequential periodic sensing for cognitive radios," IEEE J. Sel. Areas Commun., vol. 32, pp. 503-515, March 2014.

[25] A. Mariani, K. Sithamparanathan, and A. Giorgetti, "Periodic spectrum sensing with non-continuous primary user transmissions," IEEE Trans. Wireless Commun., vol. PP, no. 99, pp. 1-1, 2014.

[26] G. Ozcan, M. C. Gursoy, and S. Gezici, "Error rate analysis of cognitive radio transmissions with imperfect channel sensing," IEEE Trans. Wireless Commun., vol. 13, pp. 1642-1655, March 2014.

[27] D. T. C. Wong, S. Zheng, and Y. C. Liang, "Cognitive multi-channel MAC protocols with perfect and imperfect sensing," in 2011 IEEE International Conference on Communications (ICC), pp. 1-5, June 2011.

[28] Y. Zhang, Q. Zhang, B. Cao, and P. Chen, "Model free dynamic sensing order selection for imperfect sensing multichannel cognitive radio networks: A Q-learning approach," in Communication Systems (ICCS), 2014 IEEE International Conference on, pp. 364-368, Nov 2014.

[29] L.-C. Wang, C.-W. Wang, and F. Adachi, "Load-balancing spectrum decision for cognitive radio networks," IEEE J. Sel. Areas Commun., vol. 29, pp. 757-769, April 2011.

[30] B. Liu, Z. Li, J. Si, and F. Zhou, "Optimal sensing interval in cognitive radio networks with imperfect spectrum sensing," IET Communications, vol. 10, no. 2, pp. 189-198, 2016.

[31] Y. Chen and Z. Tang, "Effect of spectrum sensing errors on the performance of OFDM-based cognitive radio transmission," IEEE Trans. Wireless Commun., vol. 11, pp. 2342-2350, June 2012.

[32] S. Kim, T. Park, W. Choi, and Y. Han, "The capacity of cognitive ad-hoc networks with carrier sensing errors," in 2011 
6th International ICST Conference on Cognitive Radio Oriented Wireless Networks and Communications (CROWNCOM), pp. 380-384, June 2011.

[33] H. Su and X. Zhang, "Cross-layer based opportunistic MAC protocols for QoS provisionings over cognitive radio wireless networks," IEEE J. Sel. Areas Commun., vol. 26, pp. 118-129, Jan 2008.

[34] H. Kim and K. G. Shin, "Efficient discovery of spectrum opportunities with MAC-Layer sensing in cognitive radio networks," IEEE Trans. Mobile Comput., vol. 7, pp. 533-545, May 2008.

[35] B. Gao, Y. Yang, and J. M. Park, "Channel aggregation in cognitive radio networks with practical considerations," in 2011 IEEE Int. Conference on Commun. (ICC), pp. 1-5, June 2011.

[36] R. Murty, R. Chandra, T. Moscibroda, and P. Bahl, "Senseless: A database-driven white spaces network," IEEE Trans. Mobile Comput., vol. 11, pp. 189-203, Feb 2012.

[37] I. Akyildiz, W.-Y. Lee, M. C. Vuran, and S. Mohanty, "A survey on spectrum management in cognitive radio networks," IEEE Communications Magazine, vol. 46, pp. 40-48, April 2008.

[38] D. Cabric, S. Mishra, and R. Brodersen, "Implementation issues in spectrum sensing for cognitive radios," in Conference Record of the Thirty-Eighth Asilomar Conference on Signals, Systems and Computers, 2004., vol. 1, pp. 772-776 Vol.1, Nov 2004.

[39] A. Mariani, A. Giorgetti, and M. Chiani, "Effects of noise power estimation on energy detection for cognitive radio applications," IEEE Trans. Commun., vol. 59, pp. 3410-3420, December 2011.

[40] A. Mariani, A. Giorgetti, and M. Chiani, "Wideband spectrum sensing by model order selection," IEEE Trans. Wireless Commun., vol. 14, pp. 6710-6721, Dec 2015.

[41] K. Cicho, A. Kliks, and H. Bogucka, "Energy-efficient cooperative spectrum sensing: A survey," IEEE Commun. Surveys Tuts., vol. 18, pp. 1861-1886, thirdquarter 2016.

[42] A. Ali and W. Hamouda, "Advances on spectrum sensing for cognitive radio networks: Theory and applications," IEEE Communications Surveys Tutorials, vol. PP, no. 99, pp. 1-1, 2016.

[43] M. Abramowitz and I. Stegun, Handbook of Mathematical Functions, With Formulas, Graphs, and Mathematical Tables,. Dover Publications, Incorporated, 1974. 